\title{
Evaluation of a Constant Associated with a Parking Problem
}

\author{
By M. Lal and P. Gillard*
}

\begin{abstract}
A constant associated with a random space filling problem is computed to 19D. This is achieved by numerically solving an integral-difference equation.
\end{abstract}

1. Introduction. During the past few years, several mathematicians, $\mathbf{A}$. Rényi [1], A. Dvoretzky and H. Robbins [2] and J. J. A. Beenakker [3], have studied the random process in which cars of length 1 are parked in a street $[0, x]$ of length $x \geqq 1$. The centre of cars is a random variable which is uniformly distributed on $\left[\frac{1}{2}, x-\frac{1}{2}\right]$. Let $N_{x}$ denote the number of cars parked so that the distance between any two consecutive cars is less than or equal to 1 . The expectation $M(x)=E\left(N_{x}\right)$ is a continuous function for $x \geqq 1$ and satisfies the integral equation

$$
M(x+1)=\frac{2}{x} \int_{0}^{x} M(t) d t+1, \quad x>0,
$$

together with the initial conditions

$$
M(x)=0, \quad 0 \leqq x<1, \quad M(1)=1 .
$$

In the investigations [1], [2], [3], Eq. (1) has been solved to a varying degree of accuracy. These solutions are

$$
\begin{gathered}
M(x)=\lambda(x+1)-1+O\left(x^{-n}\right), \quad n \geqq 1 \quad \text { (Rényi, 1958), } \\
M(x)=\lambda(x+1)-1+O\left(\left(\frac{2 e}{x}\right)^{x-3 / 2}\right), \quad x \rightarrow \infty
\end{gathered}
$$

(Dvoretzky and Robbins, 1964),

$$
M(x)=\lambda(x+1)-1+o\left(\left(\frac{2 e}{x \log x}\right)^{x}\right), \quad x \rightarrow \infty \quad \text { (Beenakker, 1966), }
$$

where

$$
\lambda=\int_{0}^{\infty} \exp \left(2 \int_{0}^{t} \frac{\left(e^{-u}-1\right)}{u} d u\right) d t
$$

A. Palásti [4] considered the corresponding problem in the two- and three-dimen-

Received April 10, 1973.

AMS (MOS) subject classifications (1970). Primary 65R05, 65Q05; Secondary 39A10.

Key words and phrases. Differential-difference equation, random space filling.

* This work was partially supported by the National Research Council of Canada under the grant \#A-4026. 
sional space and conjectured that the corresponding constants are $\lambda^{2}$ and $\lambda^{3}$, respectively. Because of the occurrence of $\lambda$ in these three cases and possibly in the $n$-dimensional case in general, it is desirable to evaluate $\lambda$ as accurately as possible.

2. Numerical Computation of $M(x)$. For small values of $x, M(x)$ can be obtained by evaluating the integral in Eq. (1) recursively [5]. Since $M(x)=0,0 \leqq$ $x<1$, we immediately obtain $M(x)$, for the interval $1 \leqq x<2$,

$$
M(x)=\frac{2}{x} \int_{0}^{x<1} M(t) d t+1=1, \quad 1 \leqq x<2,
$$

and

$$
M(2)=2\left(\int_{0}^{1-\epsilon} M(t) d t+\int_{1-\epsilon}^{1+\epsilon} M(t) d t\right)+1=1
$$

Thus, $M(x)=1$ for $1 \leqq x \leqq 2$ and, using this in the integrand in Eq. (1), we obtain

$$
\begin{array}{ll}
M(x)=3-\frac{2}{(x-1)}, & 2 \leqq x \leqq 3, \\
M(x)=7-\frac{10+4 \ln (x-2)}{(x-1)}, & 3 \leqq x \leqq 4 .
\end{array}
$$

For $x>4$, the evaluation of successive integrals becomes tedious and a numerical method is most desirable. However, the above expressions are very useful for checking the accuracy of the numerical method to be employed.

For numerical integration of Eq. (1), we employ the recursion relation

$$
\begin{gathered}
(x+h) M(x+h+1)-x M(x+1) \\
=2 \int_{x}^{x+h} M(t) d t+h
\end{gathered}
$$

and, putting $x=0, h, 2 h, 3 h, \cdots, M(x)$ was computed for $x=(1+h)(h) 50$. The integral on the right side of Eq. (5) was approximated with a trapezoidal rule.

In order to improve the accuracy of $M(x)$, we perform four trapezoidal approximations to $M(x)$ with $h=1 / 50,1 / 100,1 / 200$, and $1 / 400$ and use these results to eliminate terms of order $h^{2}, h^{4}, h^{6}$, in the Euler-Maclaurin integration formula. Thus, we obtain Romberg extrapolations of $O\left(h^{8}\right)$ [6].

To check the over-all accuracy of the numerical integration of $M(x)$, it is essential to estimate the absolute error in Romberg extrapolation. In the present case, this is not possible since $M(x)$ has discontinuities in the derivatives at $x=1,2,3, \cdots$, and we have to rely on some practical criterion. If the last term removed in the Euler-Maclaurin formula, i.e., $h^{6}$ with $h=1 / 400$ is used as the estimate of error in $M(x)$, we obtain an error $\sim 2 \times 10^{-17}$ in $M(x)$. For $x \leqq 4$, the numerical values of $M(x)$ agreed with those obtained from the analytic expressions to 17D. All arithmetic was done with a word-length of 28 decimal digits in order to minimize the building up of round-off errors. Values of $M(x)$ for $x=1(1) 16$, accurate to 17D, are given in Table 1. 
TABLE 1

Values of $M(x)$ and $\epsilon(x)$

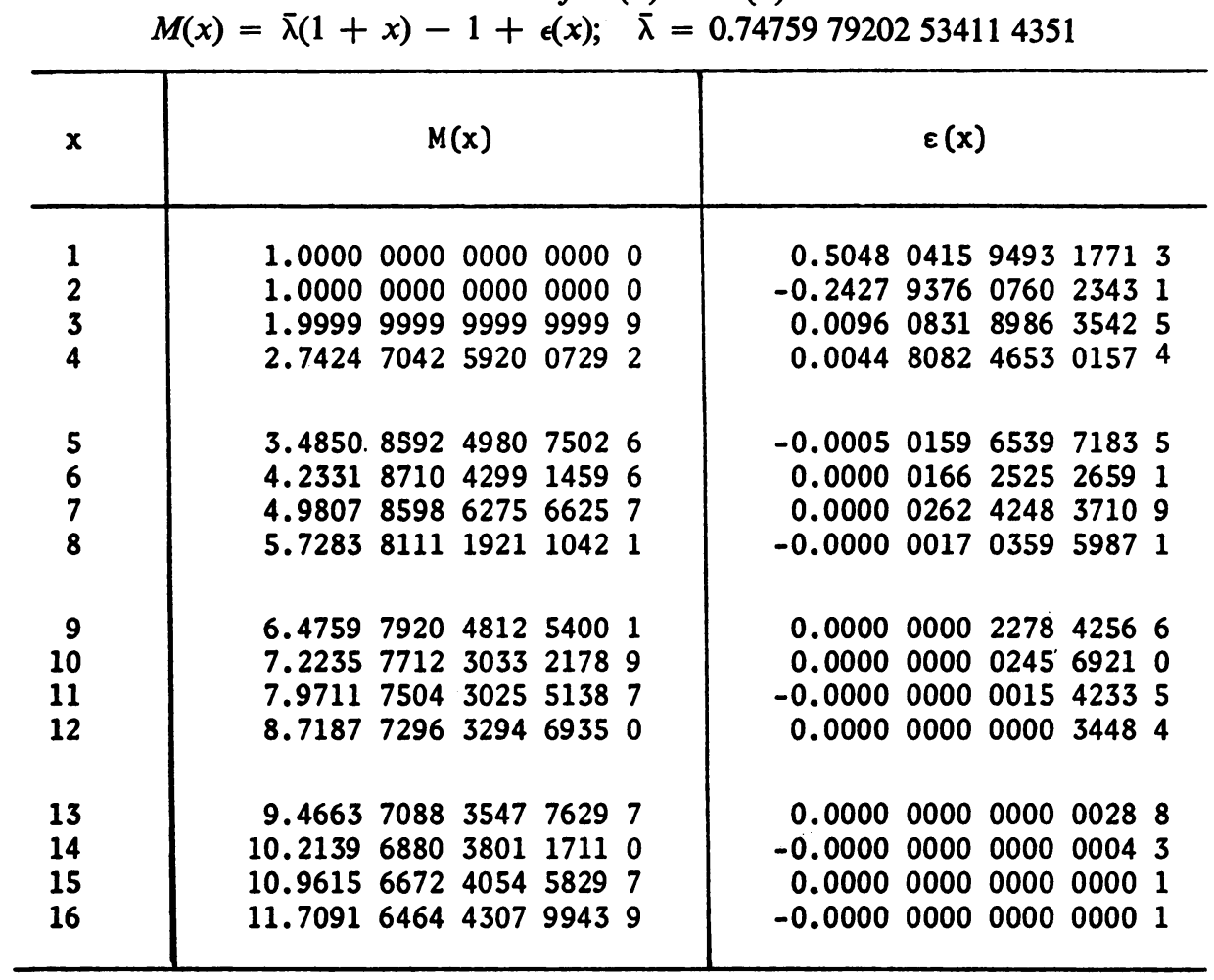

3. Evaluation of $\lambda$. Dvoretzky and Robbins [2] have shown that $\lambda$ is given by

$$
\operatorname{Inf}_{x \leq t \leq x+1} \frac{M(t)+1}{t+1} \leqq \lambda \leqq \operatorname{Sup}_{x \leq t \leq x+1} \frac{M(t)+1}{(t+1)}
$$

With the numerical values of $M(t)$ for $t=2(.02) 50$, the ratios $(M(t)+1) /(t+1)$ were computed. As $t$ is increased, the bounds for $\lambda$ are constantly narrowed. For $t \geqq 20$, the first 19 decimal digits of the ratio $(M(t)+1) /(t+1)$ remained stable. These stable digits define to 19D the accurate value $\bar{\lambda}$, of $\lambda$,

$$
\begin{aligned}
\bar{\lambda} & =\int_{0}^{\infty} \exp \left(2 \int_{0}^{t} \frac{\left(e^{-u}-1\right)}{u} d u\right) d t \\
& =0.74759,79202,53411,4351
\end{aligned}
$$

It is of interest to compute the error term $\epsilon(x)$ which appears in the expression of $M(x)$,

$$
M(x)=\bar{\lambda}(1+x)-1+\epsilon(x)
$$

Values of $\epsilon(x)$ for integral arguments are given in Table 1 . 
Acknowledgment. We wish to thank the referee for several helpful suggestions.

Added in proof. Recent computations of Lal and Tiller give $\bar{\lambda}=.74759,79202$, $53411,43517,87309,43636,52421,02617,2439$, accurate to 44D.

Department of Mathematics

Memorial University of Newfoundland

St. John's Newfoundland, Canada

1. A RÉNYI, "On a one dimensional problem concerning random space-filling," Magyar Tud. Akad. Mat. Kutató Int. Közl., v. 3, 1958, no. 1/2, pp. 109-127. (Hungarian) MR 21 \#3039.

2. A. Dvoretzky \& H. Robbins, “On the 'parking' problem," Magyar Tud. Akad. Mat. Kutató Int. Közl., v. 9, 1964, pp. 209-225. MR 30 \#3488.

3. J. J. A. BEENAKKER, The Differential-Difference Equation $\alpha x f^{\prime}(x)+f(x-1)=0$, Ph.D. Thesis, Technische Hogeschool, Eindhoven, The Netherlands, 1966.

4. I. PALÁsti, "On some random space filling problems," Magyar Tud. Akad. Mat. Kutató Int. Közl., v. 6, 1960, pp. 353-360. (Russian) MR 26 \#4466.

5. Mohan Lal \& PaUl Gillard, "Numerical solution of two differential-difference equations on analytic theory of numbers," Conference on Numerical Solution of Differential Equations, Lecture Notes in Math., vol. 109, Springer-Verlag, Berlin and New York, 1969, pp. 179-187.

6. A. Ralston \& H. S. WILf (Editors), Mathematical Methods for Digital Computers. Vol. 2, Wiley, New York, 1967, p. 135. MR 35 \#2516. 